\title{
Bioacústica como método de avaliação do comportamento em pastejo de novilhas Girolando
}

\author{
[Bioacoustics as a tool for evaluating grazing behavior of Girolando heifers] \\ H.M. Veit ${ }^{1}$, A.K.D. Salman ${ }^{2 *}$, P.G. Cruz ${ }^{2}$, E.C. Souza ${ }^{3}$, E. Schmitt ${ }^{4}$ \\ ${ }^{1}$ Programa de pós-graduação em Ciência Ambiental - Unir-Embrapa - Rolim de Moura, RO \\ ${ }^{2}$ Embrapa Rondônia - Porto Velho, RO \\ ${ }^{3}$ Programa de pós-graduação em Desenvolvimento Regional e Meio Ambiente - Unir - Porto Velho, RO \\ ${ }^{4}$ Universidade Federal de Pelotas - Pelotas, RS
}

\begin{abstract}
RESUMO
Objetivou-se validar o método de análise de dados bioacústicos na descrição do comportamento em pastejo de novilhas em área de integração lavoura-pecuária (iLP) e floresta (iLPF). Foram utilizadas oito novilhas Girolando (3/4Holandês x $1 / 4 \mathrm{Gir})$ com média de $25 \pm 6,8$ meses de idade e $268 \pm 83 \mathrm{~kg}$ de peso vivo (PV), distribuídas em delineamento crossover $2 \times 2$ (dois sistemas $\mathrm{x}$ dois períodos de avaliação). A coleta de dados para análise bioacústica foi realizada utilizando-se gravadores MP3. Esses dados foram analisados pelo software Audacity ${ }^{\circledR}$ para identificação das atividades de pastejo, ruminação e ócio. Concomitantemente, o comportamento também foi avaliado pelo método de observação visual, realizado no período de 8 até 16 horas, em intervalos de 15 minutos. Não houve diferença $(P>0,05)$ entre médias dos tempos (minutos) de pastejo, ruminação e ócio registrados pelos métodos de bioacústica e avaliação visual, sendo, respectivamente, 127 vs. 137; 122 vs. 113 e 238 vs. 216, no sistema iLP, e 134 vs. 103; 130 vs. 165 e 233 vs. 203, no sistema iLPF. Por meio desses resultados, foi possível concluir que o método da bioacústica é uma importante ferramenta para avaliar o comportamento de pastejo em diferentes sistemas de produção a pasto, com a vantagem de possibilitar o registro de atividades durante o período noturno, além de maior praticidade e precisão do que o método observacional.
\end{abstract}

Palavras-chave: ambiência, bem-estar animal, estresse térmico, sistema agroflorestal

\section{ABSTRACT}

Aiming to validate the analysis of bioacoustic data for describing grazing behavior of heifers in integrated crop, livestock (ICL) and forestry (ICLF) systems. Eight $25 \pm 6.8$ month-old Girolando $(3 / 4$ Holstein $\times 1 / 4$ Gir $)$ heifers with $268 \pm 83 \mathrm{~kg}$ of live weight $(L W)$ were distributed in $2 x 2$ crossover design (two systems $x$ two evaluation periods). Data collection for bioacoustic analysis was done with MP3 recorders. These data were analyzed by Audacity ${ }^{\circledR}$ software for identification of the activities of grazing, rumination, and resting. Concomitantly, behavior was also analyzed by the method of visual observation from 8AM to 4PM in intervals of 15 minutes. There was no difference $(P>0.05)$ between the methods of bioacustic and visual observation considering the means of time (minutes) of grazing, rumination, and resting, respectively: $127 \mathrm{vs} .137 ; 122 \mathrm{vs}$. 113; and 238 vs. 216 in ICL system and; 134 vs. 103; 130 vs. 165 and 233 vs. 203 in ICLF system. In conclusion, the bioacustic method is a tool that can be useful for evaluating bovine grazing behavior within different grazing systems, with the advantage of allowing report of activities during the night with higher practicality and precision than the visual observation method.

Keywords: ambience, animal welfare, thermal stress, agroforestry system

\section{INTRODUÇÃO}

O estudo do comportamento em pastejo é importante para se conhecer a relação da interface planta-animal (Mezzalira et al., 2011), sendo o desempenho animal determinado pela

Recebido em 15 de setembro de 2016

Aceito em 18 de outubro de 2017

* Autor para correspondência (corresponding author)

E-mail: ana.salman@embrapa.br quantidade e qualidade da forragem consumida (Santana Júnior et al., 2013). Mediante o estudo do comportamento dos animais, é possível apresentar soluções para problemas relacionados com a redução do consumo em épocas críticas para produção de leite (Albrigth, 1993). Os resultados de pesquisas podem descrever os principais problemas que limitam a 
produtividade e o bem-estar animal de vacas lactantes e podem ser utilizados para orientar extensionistas, pesquisadores e formuladores de políticas públicas (Costa et al., 2013). Dessa forma, medidas nutricionais ou de manejo podem ser aplicadas na melhoria produtiva nos sistemas de produção a pasto, tanto de explorações de corte como de leite.

Carvalho et al. (2007) citam várias técnicas ou equipamentos para o estudo do comportamento de bovinos em pastejo. Entretanto, a principal metodologia utilizada em estudos de avaliação do comportamento de bovinos em pastagem é a observação visual (Fischer et al., 1998; Salla et al., 2003; Olivo et al., 2006; Baggio et al., 2008; Silva et al., 2009; Oliveira et al., 2011; Lopes et al., 2016). Essa técnica consiste no registro do comportamento em pastejo do animal em intervalos de tempo predeterminados, com o auxílio de binóculos para melhor observação das atividades realizadas por eles. No entanto, a avaliação visual apresenta algumas limitações, tais como dificuldade de avaliações comportamentais no período noturno, áreas extensas de pastagem com a presença de obstáculos para visualização, possibilidade de interferência dos observadores no comportamento dos animais e registro incorreto de atividades por observadores sem treinamento prévio.

Uma alternativa para o estudo do comportamento em pastejo é o método da bioacústica, que consiste no registro dos sons produzidos pelos animais em pastejo, gerando áudios com características acústicas discriminadas em frequência $(\mathrm{Hz})$, intensidade $(\mathrm{dB})$, duração $(\mathrm{s})$ e intervalos de tempo (s) (Trindade et al., 2011). A bioacústica é um método não invasivo, com baixo custo, que possibilita a identificação de atividades dos ruminantes de forma contínua, sem afetar o comportamento do animal. O princípio da metodologia consiste em identificar, pelos padrões de sons, as atividades de pastejo, ruminação e ócio dos animais em intervalos longos e ininterruptos. Outra possibilidade do método está relacionada à intensidade e ao tipo de ondas sonoras produzidas pelo pastejo que podem ser associadas com a quantidade de forragem ingerida pelos animais (Nelson et al., 2005; Carvalho et al., 2009).

Diante do exposto, o presente trabalho teve como objetivo validar o método de bioacústica em relação ao método de avaliação visual para caracterização do comportamento em pastejo de novilhas Girolando, em sistema de integração lavoura-pecuária (iLP) e floresta (iLPF).

\section{MATERIAL E MÉTODOS}

A pesquisa foi conduzida no campo experimental da Empresa Brasileira de Pesquisa Agropecuária - Embrapa, localizada no município de Porto Velho, Rondônia, Brasil, com as coordenadas geográficas $8^{\circ} 48^{\prime} 03.89^{\prime}$ S e 6350'53.08” O. O clima, segundo o sistema de classificação de Köppen, é do tipo Aw, tropical chuvoso, com estação relativamente seca durante o ano e temperaturas médias anuais de $25,5^{\circ} \mathrm{C}$. O regime pluviométrico é caracterizado por período chuvoso, com média anual próxima de 2.400 $\mathrm{mm}$, que está compreendido entre os meses de novembro a abril, e período seco, entre os meses de maio e setembro.

O experimento teve 60 dias de duração, com início em setembro de 2015 e término em novembro de 2015. Foram utilizadas oito novilhas Girolando ( $3 / 4$ Holandês x $1 / 4 \mathrm{Gir})$, com média de idade de $25 \pm 6,8$ meses e $268 \pm 83 \mathrm{~kg}$ de peso vivo, divididas em dois grupos homogêneos para idade, grau de sangue e peso. Os animais experimentais foram mantidos em pastagem de capim-xaraés (Brachiaria brizantha cv. Xaraés).

Em ambos os sistemas, a área de pastagem era de cinco hectares, dividida em quatro piquetes de 1,25ha, com praça de alimentação localizada no centro de cada sistema, contendo bebedouro e cocho, onde os animais receberam suplementação mineral e água ad libitum. O sistema iLPF estava sombreado por árvores de eucalipto plantadas em março de 2013, em renques com quatro linhas em espaçamento $3 \times 3 \mathrm{~m}$. No período de realização do estudo, as árvores apresentavam, em média, $11,9 \mathrm{~cm}$ de diâmetro e $13,8 \mathrm{~m}$ de altura total, sendo a média de cobertura de copa de $65 \%$. A pastagem foi manejada com lotação intermitente, com 10 dias de ocupação e 30 dias de descanso, com oferta de 41,9 e $32,3 \mathrm{~kg}$ de $\mathrm{MS} / 100 \mathrm{~kg}$ de peso vivo (PV), nos sistemas iLP e iLPF, respectivamente, e lotação média de 2,5UA/ha. Na Tab. 1, encontram-se os valores de matéria seca (MS), fibra em detergente ácido (FDA), fibra em detergente neutro (FDN), lignina (LIG) e proteína bruta $(\mathrm{PB})$ do capim-xaraés nos sistemas iLP e iLPF. 
Tabela 1. Composição químico-bromatológica do capim-xaraés (Brachiaria brizantha cv. Xaraés) nos sistemas de integração lavoura-pecuária (iLP) e floresta (iLPF)

\begin{tabular}{cccccc}
\hline Sistema & MS & PB & FDN & FDA & LIG \\
\hline & $(\%)$ & \multicolumn{3}{c}{$\%$ na MS } & \\
\hline iLP & 30,6 & 9,5 & 59,9 & 27,5 & 2,2 \\
iLPF & 25,9 & 12,5 & 60,2 & 28,8 & 2,5 \\
\hline
\end{tabular}

MS - matéria seca, PB - proteína bruta, FDN - fibra em detergente neutro, FDA - fibra em detergente ácido, LIG lignina.

O delineamento experimental utilizado foi $\mathrm{o}$ crossover $2 \times 2$, considerando dois sistemas de integração (iLP e iLPF) e dois períodos experimentais (Fig. 1). Cada período experimental compreendeu 10 dias de adaptação, seguidos de 20 dias para coleta de dados, com a troca de grupos de animais entre os sistemas no final do primeiro período experimental.

\section{Período experimental de 60 dias}

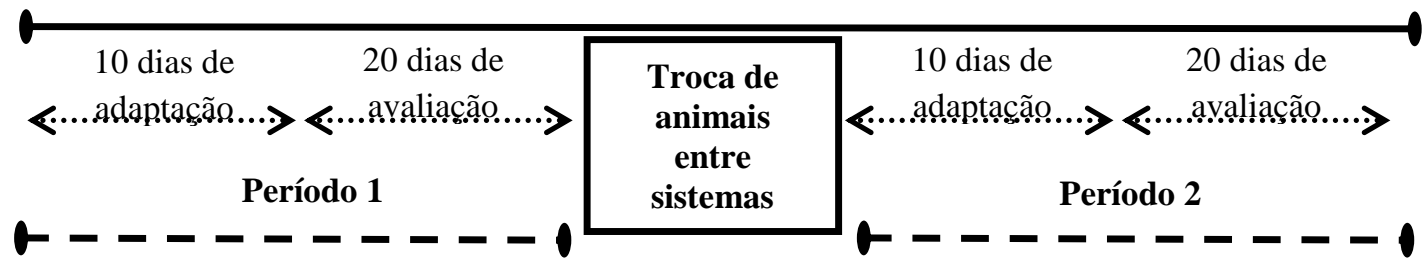

Figura 1. Esquema representativo do delineamento crossover $2 \times 2$.

Os dados de bioacústica foram coletados com gravadores de áudio adaptados em cabrestos por 48h (no quinto e no sexto dia de ocupação de um piquete escolhido ao acaso). Após esse período, os áudios foram analisados com o auxílio do programa Audacity®, o qual gera espectrogramas específicos (Figura 2) que permitem a identificação dos tempos gastos com as atividades de pastejo (Figura 2A), ruminação e ócio (Figura 2B).

A avaliação visual de comportamento foi realizada durante o primeiro dia de coleta de dados de bioacústica (quinto dia de ocupação do piquete), por duplas de observadores, no período de oito até 16 horas, com observações feitas em intervalos de 15 minutos. Os animais experimentais foram numerados com tinta óleo nos flancos. As atividades observadas (pastejo, ruminação e ócio) foram registradas em planilhas para posterior cálculo de tempo gasto em cada atividade.

Para comparação do método de bioacústica com o de avaliação visual, foi considerado somente o tempo de registro de oito até 16 horas do primeiro dia de avaliação.

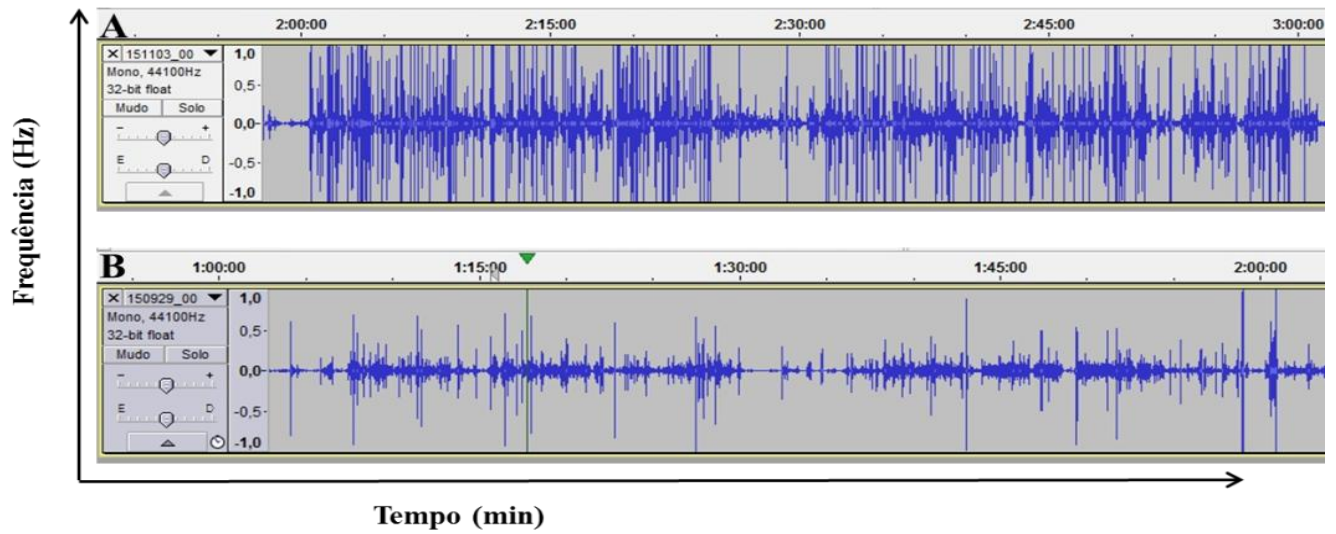

Figura 2. Espectrogramas específicos gerados a partir dos sons das atividades de pastejo (A) e ócio (B). 
A análise de variância no delineamento crossover $2 \times 2$ foi realizada utilizando-se $\mathrm{o}$ procedimento MIXED do SAS (Statistical Analysis System), conforme o modelo abaixo:

em que:

$$
\mathrm{Y}_{\mathrm{ijklm}}=\mu+\mathrm{S}_{\mathrm{ij}}+\mathrm{P}_{\mathrm{k}}+\mathrm{T}_{1}+\mathrm{H}_{\mathrm{m}}+\mathrm{TH}_{\mathrm{lm}}+e_{i j k l m}
$$

$\mathrm{Y}_{\mathrm{ijklm}}$ : variável resposta;

$\mu$ : média geral;

S: efeito do i-ésimo indivíduo (1 a 8) na j-ésima sequência (1 e 2);

P: efeito fixo do k-ésimo período (1 e 2);

T: efeito fixo do l-ésimo sistema de produção (iLP e iLPF);

$\mathrm{H}$ : efeito fixo do m-ésimo horário (diurno e noturno);

$e_{\mathrm{ijklm}}$ : erro aleatório.

As médias dos tempos despendidos em cada atividade foram comparadas pelo teste de TukeyKramer a 5\% de significância.

\section{RESULTADOS E DISCUSSÃO}

As médias dos tempos despendidos com as atividades de pastejo, ruminação e ócio nos sistemas iLP e iLPF não apresentaram diferença $(\mathrm{P}>0,05)$ em relação aos métodos de avaliação utilizados (Tab. 2).

Como não houve interação entre o método de avaliação e os sistemas de integração iLP e iLPF (Tab. 2), foi possível fazer a comparação entre os métodos de bioacústica e de avaliação visual considerando-se as médias dos tempos gastos por novilhas Girolando em ambos os sistemas para realização das atividades de pastejo, ruminação e ócio (Fig. 3).

Considerando-se o tempo total de coleta de dados (96 horas) pelo método da bioacústica
(Tab. 3), a atividade de pastejo apresentou diferença $(\mathrm{P}<0,05)$ entre os dois sistemas de integração. Essa diferença não foi observada pelo método de avaliação visual, o que caracteriza maior precisão da bioacústica na avaliação do tempo de pastejo das novilhas.

Os métodos da bioacústica e de avaliação visual não apresentaram diferença $(\mathrm{P}>0,05)$, considerando-se as médias de tempo de pastejo, ruminação e ócio no período de oito a 16 horas. A bioacústica se caracteriza como metodologia de avaliação da atividade de comportamento de bovinos em pastejo pouco invasiva por permitir registrar as atividades realizadas pelos animais sem que eles sofram interferência em seu comportamento por ação do homem (Trindade $e t$ al., 2011). Ainda possui como características o baixo custo para execução (Nelson et al., 2005), a economia de mão de obra e a possibilidade de registros noturnos com maior facilidade.

Tabela 2. Comparação entre o método de bioacústica e de avaliação visual no horário compreendido entre oito e 16 horas, considerando-se as médias dos tempos gastos (minutos) por novilhas Girolando para realizar as atividades de pastejo, ruminação e ócio em sistemas iLP e iLPF

\begin{tabular}{|c|c|c|c|c|}
\hline Atividade & Método & Sistema & Tempo (minutos) & Erro-padrão \\
\hline \multirow{4}{*}{ Pastejo } & iLP & Bioacústica & 127 & $\pm 19,72$ \\
\hline & & Visual & 137 & $\pm 19,72$ \\
\hline & iLPF & Bioacústica & 134 & $\pm 21,04$ \\
\hline & & Visual & 103 & $\pm 19,72$ \\
\hline \multirow{4}{*}{ Ruminação } & iLP & Bioacústica & 122 & $\pm 15,10$ \\
\hline & & Visual & 113 & $\pm 16,21$ \\
\hline & iLPF & Bioacústica & 130 & $\pm 15,10$ \\
\hline & & Visual & 165 & $\pm 15,10$ \\
\hline \multirow{4}{*}{ Ócio } & iLP & Bioacústica & 238 & $\pm 15,03$ \\
\hline & & Visual & 216 & $\pm 15,95$ \\
\hline & iLPF & Bioacústica & 233 & $\pm 15,03$ \\
\hline & & Visual & 202 & $\pm 15,03$ \\
\hline
\end{tabular}

Médias seguidas de letras diferentes na coluna diferem entre si pelo teste Tukey-Kramer a 5\% de significância. 
Bioacústica como método...
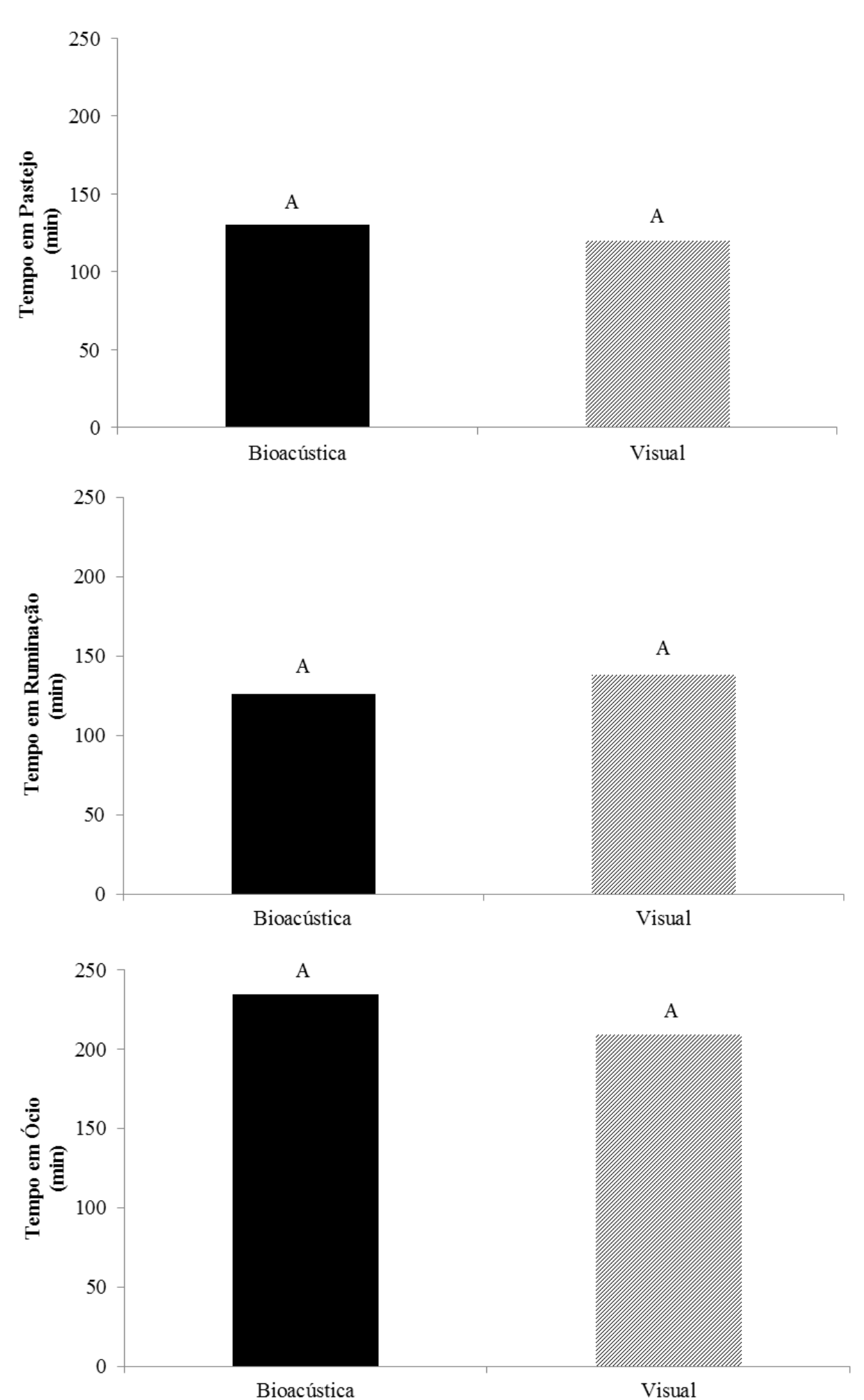

Figura 3. Tempos gastos por novilhas Girolando em atividades de pastejo, ruminação e ócio, obtidos por avaliação bioacústica (ロ) ou observação visual (圈) (P>0,05). 
Tabela 3. Tempo gasto em pastejo (minutos) por novilhas Girolando, coletado pelo método bioacústica durante as $96 \mathrm{~h}$ de coleta de dados (48h em cada período), nos horários diurno e noturno, nos sistemas iLP e iLPF

\begin{tabular}{cccc} 
Horário & Sistema & Tempo & Pr \\
\hline \multirow{2}{*}{ Diurno } & iLP & $437 \mathrm{~B}$ & \\
& iLPF & $581 \mathrm{~A}$ & \\
\multirow{2}{*}{ Noturno } & iLP & $228 \mathrm{C}$ & 0,0083 \\
& iLPF & 238 C & \\
\hline
\end{tabular}

Médias seguidas de letras iguais na coluna não se diferenciam pelo teste de Tukey $(\mathrm{P}<0,05)$.

A literatura apresenta vários trabalhos realizados com o objetivo de aprimorar a metodologia de avaliação visual, considerando-se diferentes intervalos entre as observações para registro das atividades realizadas pelos animais, variando de cinco, 10,15 e 30 minutos. Segundo Oliveira et al. (2011), os intervalos de cinco minutos são mais indicados, pois intervalos maiores podem subestimar os resultados. Silva et al. (2008) encontraram diferenças ao avaliarem intervalos de 10,20 e 30 minutos, concluindo que intervalos de até 30 minutos podem ser utilizados para avaliar tempo total de atividade diária, porém recomendam intervalos de, no máximo, 10 minutos para séries discretas de atividades.

Com a bioacústica, é possível obter registros contínuos, com o tempo variando de acordo com a capacidade de armazenamento e durabilidade da bateria do gravador. Com isso, eleva-se a precisão das informações por se eliminar o intervalo entre observações, quando comparado com a observação visual de comportamento dos animais, além de eliminar os problemas com a observação no período noturno (Forbes, 1986). A avaliação no horário noturno com a metodologia visual apresenta algumas limitações, principalmente relacionadas à iluminação artificial, que pode interferir no comportamento dos animais.

Dessa forma, a bioacústica mostrou-se potencialmente mais eficaz para identificar as alterações comportamentais conforme sistema de criação ou ambiente em que os animais são expostos, tal qual em situações de estresse térmico (Silva, 2000). Neste estudo, os resultados mostraram a possibilidade de se identificarem as alterações relacionadas ao horário do dia em que acontecem o pastejo, a ruminação e o ócio, assim como o tempo exato despendido nessas atividades de acordo com o sistema de produção.

Durante a avaliação dos dados bioacústicos também é possível estimar a frequência respiratória dos animais em pastejo (número de respirações por minuto), o que é de grande importância para determinação do nível de estresse térmico segundo o índice de Benezra, que utiliza temperatura retal (TR) e frequência respiratória (FR). Também é possível realizar o cálculo da taxa de bocados e avaliar o consumo realizado pelos animais, como apresentado nos trabalhos de Galii et al. (2011) e Ungar e Rutter (2006). Isso porque o movimento do bocado, composto pela apreensão e pelo corte do capim, apresenta-se de forma distinta nos áudios.

As principais desvantagens do método da bioacústica são o tempo limitado de gravação de áudio, já que a fonte de energia dos gravadores utilizados são pilhas alcalinas, as quais não possuem tempo de duração maior que 48 horas. Além disso, há risco de perda de registro das atividades no caso de os gravadores serem danificados em brigas entre animais ou quando os animais se coçam em árvores ou cercas. Outra desvantagem é com o fato de não ser possível distinguir o posicionamento em pé ou deitado dos animais quando estes estão realizando as atividades de ócio e de ruminação na pastagem; ou o posicionamento ao sol e à sombra quando as avaliações são realizadas em pastagens sombreadas. Essas informações são importantes porque estão relacionadas com a condição de conforto térmico dos animais. Segundo Damasceno et al. (1999), em ambientes com temperatura elevada, os animais tendem a ruminar maior tempo em pé. Um outro exemplo foi demonstrado por Camargo (1988) que observou que, para atividade de ócio durante o dia, os animais, nas horas de maior temperatura ambiente, preferem ficar em ócio em pé e, no período noturno, tendem a realizar o ócio deitados.

\section{CONCLUSÃO}

O método de bioacústica foi eficiente na avaliação do comportamento em pastejo de novilhas Girolando em sistema de integração 
lavoura-pecuária (iLP) e floresta (iLPF), pois permitiu o registro das atividades nos períodos diurno e noturno. Esse método permite registro mais preciso e por período contínuo das atividades de pastejo, ruminação e ócio, o que diminui a possibilidade de subestimação ou superestimação dos resultados.

\section{AGRADECIMENTOS}

Ao Conselho Nacional de Desenvolvimento Científico e Tecnológico $(\mathrm{CNPq})$ e à Fundação Rondônia de Amparo ao Desenvolvimento das Ações Científicas e Tecnológicas e à Pesquisa do Estado de Rondônia (Fapero), pelo apoio financeiro.

\section{REFERÊNCIAS}

ALBRIGHT, J.L. Nutrition, feeding and calves: feeding behaviour of dairy cattle. J. Dairy Sci., v.76, p.485-498, 1993.

BAGGiO, C.; CARVALHO, P.C.R.; SILVA, J.L.S. et al. Padrões de uso do tempo por novilhos em pastagem consorciada de azevém anual e aveia-preta. Rev. Bras. Zootec., v.37, p.1912-1918, 2008.

CAMARGO, A.C. Comportamento de vacas da raça holandês em confinamento do tipo "free stall”, no Brasil Central. 1988. 146f. Dissertação (Mestrado em Zootecnia) - Curso de Pósgraduação em Zootecnia, Fundação de Estudos Agrários Luiz de Queiroz, SP.

CARVALHO, P.C.F.; KOZLOSKI, G.V.; RIBEIRO FILHO, H.M.N. et al. Avanços metodológicos na determinação do consumo de ruminantes em pastejo. Rev. Bras. Zootec., v.36, Supl. Esp., p.151-170, 2007.

CARVALHO, P.C.F.; TRINDADE, J.K.; MEZALLIRA, J.C. et al. Do bocado ao pastejo de precisão: compreendendo a interface plantaanimal para explorar a multi-funcionalidade das pastagens. Rev. Bras. Zootec., v.38, Supl. Esp., p.109-122, 2009.

COSTA, J.H.C.; HÖTZEL, M.J.; LONGO, C.; BALCÃO, L.F.A survey of management practices that influence production and welfare of dairy cattle on family farms in southern Brazil. J. Dairy Sci., v.96, p.307-317, 2013.
DAMASCENO, J.C.; BACCARI JÚNIOR, F.; TARGA, L.A. Respostas comportamentais de vacas holandesas, com acesso à sombra constante ou limitada. Pesqui. Agropecu. Bras., v.34, p.709-715, 1999.

FISCHER, V.; DESWYSEN, A.G.; DÈSPRES, L. et al. Padrões nectemerais do comportamento ingestivo de ovinos. Rev. Bras. Zootec., v.27, p.362-369, 1998.

FORBES, J.M. The voluntary food intake of farm animals. Londres: Butterworth, 1986. 206p.

GALLI, J.R.; CANGIANO, C.A.; MILONE, D.H.; LACA, E.A. Acoustic monitoring of shortterm ingestive behavior and intake in grazing sheep. Livest. Sci., v.140, p.32-41, 2011.

LOPES, L.B.; ECKSTEIN, C.; PINA, D.S.; CARNEVALLI, R.A. The influence of trees on the thermal environment and behaviour of grazing heifers in Brazilian Midwest. Trop. Anim. Health Prod., v.48, p.755-761, 2016.

MEZZALIRA, J.C.; CARVALHO, P.C.F.; FONSECA, L. et al. Aspectos metodológicos do comportamento ingestivo de bovinos em pastejo. Rev. Bras. Zootec., v.40. p.1114-1120, 2011.

NELSON, D.E.; ALKON, P.U.; KRAUSMAN, P.R. Using acoustic telemetry to monitor foraging by penned mule deer. Wildl. Soc. B, v.33, p.624-632, 2005.

OLIVEIRA, P.A.; MARQUES, J.A.; BARBOSA, L.P. et al. Aspectos metodológicos do comportamento ingestivo de vacas lactantes em pastejo de Brachiaria decumbens. Rev. Bras. Saúde Prod. Anim., v.12, p.166-175, 2011.

OLIVO, C.J.; CHARÃO, P.S.; ZIECH, M.F. et al. Comportamento de vacas em lactação em pastagem manejada sob princípios agroecológicos. Rev. Bras. Zootec., v.35, p.24432450, 2006.

SALLA, L.E.; FISCHER, V.; FERREIRA, E.X. et al. Comportamento ingestivo de vacas jersey alimentadas com dietas contendo diferentes fontes de gordura nos primeiros 100 dias de lactação. Rev. Bras. Zootec., v.32, p.683-689, 2003.

SANTANA JUNIOR, H.A.; SILVA, R.R.; CARVALHO, G.G.P. et al. Correlação entre desempenho e comportamento ingestivo de novilhas suplementadas a pasto. Semin. Ciênc. Agrár., Londrina, v.34, p.367-376, 2013. 
SILVA, É.C.L.; MODESTO, E.C.; AZEVEDO, M. et al. Efeitos da disponibilidade de sombra sobre o desempenho, atividades comportamentais e parâmetros fisiológicos de vacas da raça Pitangueiras. Acta Sci. Anim. Sci., v.31, p.295-302, 2009.

SILVA, R.G. Introdução á bioclimatologia animal. São Paulo: Nobel, 2000. 288p.

SILVA, R.R.; PRADO, I.N.; CARVALHO, G.G.P. et al. Efeito da utilização de três intervalos de observações sobre a precisão dos resultados obtidos no estudo do comportamento ingestivo de vacas leiteiras em pastejo. Ciênc. Anim. Bras., v.9, p.319-326, 2008.
TRINDADE， J.K.; CARVALHO, P.C.F.; NEVES, F.P. et al. Notas científicas potencial de um método acústico em quantificar as atividades de bovinos em pastejo. Pesqui. Agropecu. Bras., v.46, p.965-968, 2011.

UNGAR, E.D.; RUTTER, S.M. Classifying cattle jaw movements: comparing IGER behaviour recorder and acoustic techniques. Appl. Anim. Behav. Sci., v.98, p.11-27, 2006. 